\title{
Claves de la Organización Escolar para el desarrollo de las competencias del siglo XXI
}

\author{
Clues to school organization for the development of students' \\ skills in 21st century \\ Levis Villadiego-Sánchez
}

Universidad Metropolitana de Ciencia y Tecnología. Ciudad de Panamá, Panamá

Recibido: 05/08/2021 Revisado: 20/09/2021 Aceptado: 21/12/2021 Publicado: 15/01/2022

\section{RESUMEN}

El presente artículo, desarrolla los elementos conceptuales que sustentan los componentes relevantes de la organización escolar para desarrollar las competencias en los estudiantes en respuesta a las demandas de la sociedad de la información, el conocimiento y el aprendizaje. Se plantea diferentes estrategias que operacionalizan los fundamentos atendiendo al nuevo enfoque del contrato social CTS (Ciencia, Tecnología y Sociedad), para superar el antiguo paradigma positivista y sentándola sobre un enfoque que atiende no solo los intereses de la ciencia, sino las necesidades y problemas de los contextos sociales, lo cual amplía su pertinencia, extensión y aplicabilidad. Desarrolla inicialmente, la necesidad de priorizar sobre el diseño, implementación y evaluación de las políticas públicas, que constituyen la guía u orientación de la educación, luego la necesidad de reestructurar los diseños curriculares para que respondan a los intereses, necesidades que la sociedad considere pertinentes, dándole relevancia a la implementación de estrategias que potencialicen el aprendizaje en red, y las competencias para aprender a aprender en un medio altamente cambiante, finalmente analiza las implicaciones del desarrollo profesional docente y su impacto en una educación de calidad al amparo de una sociedad marcada por los avances en materia tecnológica.

Palabras clave: Competencia, planeación, didáctica, virtual.

\begin{abstract}
This article develops the conceptual elements that support the relevant components of school organization to develop competencies in students in response to the demands of the information, knowledge and learning society. Different strategies are proposed that operates the bases according to the new approach of the social agreement CTS (Science, Technology and Society) to get over the old positivist model and set it on an approach that deals not only with the interest of science, but also the needs and the problems of social context, this situation enlarge its relevance, extension and applicability. It develops initially the need to prioritize over the design, implementation


and the review of the public policies which constitute the guide or orientation of education, later the need to restructure the curricular designs to answer to the interest, needs that society considers relevant giving the importance to the implementation of strategies that reinforce learning on net and the competences to learn how to learn in an highly changing environment. Finally, it analyzes the implications of the teacher professional development and its impact on an education with quality under the protection of a society pronounced by advances in the technological field. Keywords: Competence, planning, didactics, virtual.

\section{INTRODUCCIÓN}

Ante el panorama mundial que evidencia el impacto de las políticas neoliberales con enfoque de desarrollo económico, a través del aumento de los conflictos (entre y al interior de algunos países) y la brecha de desigualdad, la alarmante crisis humanitaria mundial generada por la violencia, la pobreza y el hambre, la creciente violación a los Derechos Humanos y el deterioro ambiental que ha puesto en jaque la sobrevivencia del ser humano, la educación enfrenta el reto de promover el desarrollo de competencias que le permita a los individuos desenvolverse de manera eficiente y eficaz en un medio que cambia aceleradamente.

Además, frente a los retos que le imprime la sociedad del conocimiento a la escuela para desarrollar competencias en los estudiantes, de tal forma que favorezca el aprender a aprender, desarrollar la creatividad e innovación, se plantea el interrogante ¿Cómo pueden organizarse las escuelas para promover entre los estudiantes las competencias del siglo XXI?

A través del artículo, se desarrollan las ideas que sustentan los diferentes elementos que debe tener la escuela, al organizarse, para desarrollar las competencias en los estudiantes que garanticen la pertinencia y calidad de los aprendizajes requeridos en la sociedad del conocimiento y el aprendizaje. Inicialmente se aborda el currículo como eje que engrana la propuesta educativa plasmada en la política educativa, la cual atien- de al nuevo enfoque del contrato social de las Ciencias, Tecnología y Sociedad; a continuación, se desarrolla la variable planeación didáctica y práctica áulica eficiente y contextual, luego, la variable desarrollo del capital humano a través de la formación continua, seguidamente y finalmente se aborda el impacto de la diada docencia-investigación en la transformación de las prácticas educativas.

Se concluye además, la importancia del currículo contextual, atendiendo a los intereses y necesidades explícitos en los diferentes Proyectos Educativos Institucionales, el cual necesita integrar las Tecnologías de la Información y las Comunicaciones en ambientes de aprendizaje enriquecidos, retantes, fruto de la acción educativa pedagógica y didácticamente intencional, fruto de un docente en formación continua.

\section{DESARROLLO}

La respuesta al interrogante inicial ¿Cómo pueden organizarse las escuelas para promover entre los estudiantes las competencias del siglo XXI? involucra diversidad de variables, destacando fundamentalmente al currículo, como eje articulador de la política educativa, la teoría pedagógica y la práctica de aula, en tanto su carácter polisémico, pone de manifiesto las diferentes concepciones en su dinámica de construcción y reconstrucción cultural, la cual responde a las características del momento histórico que se presenta. Por consiguiente, se puede plantear 
que la función esencial del currículo es hacer tangible la concepción de educación desde el marco teleológico, el cual le da sentido y autenticidad al proceso educativo y posibilita, además, las transformaciones necesarias para alcanzar la concreción de la propuesta pedagógica que contiene la visión de la educación.

En este mismo sentido, la pedagogía, la didáctica, el currículo y la evaluación en el quehacer formativo, se articulan en función de las convicciones, ideales y fundamentos compartidos que identifican las propuestas educativas con las que las comunidades se sienten representadas en los Proyectos Educativos Institucionales, esto es a través de la coherencia entre las prácticas institucionales en el desarrollo curricular y los lineamientos trazados en dichos proyectos, en consonancia con lo que plantea Santiváñez Limas (2012) "el currículo concreta los planteamientos de la concepción educativa que un país desea alcanzar" (p.34), en consecuencia, el currículo es una construcción cultural, entendido desde los contextos reales en que que se desarrolla la enseñanza y el aprendizaje, y desde la perspectiva de sus actores, la cual contiene las decisiones necesarias para planear, regular los contenidos, poner en marcha el proceso de enseñanza - aprendizaje, y la acción didáctico pedagógica del docente (Sacristán, 2010), esto es, sirve de guía que orienta estratégicamente el proceso educativo.

Desde otro punto de vista, al abordar los aspectos que describen la manera en que se debería ejercer la labor educativa en la sociedad globalizada e interconectada, se destaca la formación continua, como elemento que garantiza la supervivencia de los docentes en un medio que avanza a pasos agigantados; en concordancia con lo planteado por Pastrana
Armínola y Oviedo (2014), se trata de "prepararse o alfabetizarse en los nuevos saberes, lenguajes y tecnologías emergentes" (p. 7).

Desde este ángulo, se exige una motivación interna, comprometida, útil, de tal manera que en el docente se posibilite no solo aprender, sino desaprender y aprender a prender; lo que implica en cierta forma ganar vigencia, credibilidad y prestigio social, poniendo en escena y validando lo aprendido desde las prácticas áulicas, traducidas en nuevas y mejores maneras de enseñar, prácticas exitosas, eficientes y eficaces; aprendizajes duraderos y pertinentes.

Se trata de una práctica de aula reflexiva, orientada, consciente y responsable; sustentada en bases sólidas del conocimiento filosófico, epistemológico y pedagógico de la educación. Este aspecto sugiere un esfuerzo extra para los profesionales que no somos nativos digitales, pues se vuelve imperativo despojarse de los elementos estáticos del paradigma tradicional que fundamentó nuestro proceso de formación, hacia la búsqueda de elementos asociados al paradigma constructivista, conectivista, en medio del auge de las Tecnologías de la Información y las Comunicaciones (TIC).

En segundo lugar, es posible abordar la investigación educativa, como factor asociado al fortalecimiento de la enseñanza-aprendizaje y de la generación de conocimiento, por lo que se asume un rol activo en materia de encontrar soluciones a las problemáticas contextuales en los que se desarrollan los Proyectos Educativos; contribuyendo así al desarrollo social.

En este nuevo escenario social, el rol que asume el docente pasa de posibilitar el aprendizaje a través de entornos mediados por las TIC, a ser diseñador y generador de recur- 
sos educativos en ambientes de aprendizaje significativos (UNESCO, 2013). De igual forma, genera los espacios para potencializar el trabajo colaborativo, acerca a las personas de diferentes nacionalidades, promoviendo así la interculturalidad en Ambientes Virtuales de Aprendizaje (AVA) mediados por Objetos Virtuales de Aprendizaje (OVA), lo cual enriquece las situaciones de aprendizaje, favorece la autogestión del conocimiento y la innovación.

Desde esta óptica, cobra relevancia el sentido educativo de las Tecnologías del Aprendizaje en Red (TAR), puesto que propicia el aprendizaje a lo largo de la vida y desde diferentes contextos, relacionado con el aprendizaje sin costuras al que se refiere Cesar Coll; es decir, modifica la ecología del aprendizaje en relación con los contenidos, las herramientas y los entornos. Los diseños curriculares bajo esta modalidad educativa, son planeados desde las necesidades particulares de los individuos y sus ritmos de aprendizaje, articulando los saberes y competencias a nivel interdisciplinar, se potencializa la búsqueda de información a través de diferentes fuentes, se privilegia el análisis de la información para diferenciar lo verdaderamente importante y el trabajo colaborativo toma un valor trascendente al debatir ideas, redactar ensayos, ponencias, artículos y presentar la información a través de mapas mentales, conceptuales y cuadros comparativos, entre otros.

En la escuela, el desarrollo de competencia en entornos virtuales, aprendizaje remoto o en la modalidad de alternancia, es un proceso que poco había avanzado, se había postergado, en algunos casos debido a la falta de dotación en recursos tecnológicos e internet y de articulación con los procesos de formación del profesorado, pero ante la pandemia del
COVID-19 se han evidenciado avances significativos al respecto, los cuales deben ser garantes para seguir en esa línea e ir avanzando.

Con respecto a la función de las teorías y formas de organización curricular en los procesos de enseñanza y aprendizaje, éstas fundamentan, direccionan y organizan de manera científica y práctica la actividad educacional, evitando así la improvisación. Del mismo modo se plantea que el docente adecúa el diseño curricular a las necesidades específicas de aula en la que desarrolla su práctica educativa (Fonseca Pérez \& Gamboa Graus, 2017), por lo que resulta importante que el docente a lo largo de su formación, conozca el sustento teórico de las diferentes teorías, de tal forma que pueda interpretar, aplicar y reflexionar sobre los fundamentos del desarrollo de competencias y direccionar así, intencionalmente su práctica de aula.

De este punto de vista emerge el primer elemento indispensable para el desarrollo de competencias, un profundo conocimiento de las características individuales, potencialidades y limitaciones del sujeto que se educa, en el marco de su contexto cercano, para poder entender y traducir las necesidades de la realidad del individuo, en la planeación consciente, responsable y pertinente del acto educativo.

El contexto de la educación virtual tiene características muy particulares, esto es, promueve el trabajo colaborativo, acerca a las personas de diferentes nacionalidades, fomentando así la interculturalidad en Ambientes Virtuales de Aprendizaje (AVA) mediados por Objetos Virtuales de Aprendizaje (OVA), lo cual enriquece las situaciones de aprendizaje en procura de la autogestión del conocimiento. 
Aprender en el contexto desafiante de la pandemia del COVID-19

Ante la crisis de salud generada por el avance acelerado de la pandemia del COVID 19 y de acuerdo a las medidas establecidas por la Organización Mundial de la Salud, en materia de confinamiento y distanciamiento social, las instituciones escolares optaron por cerrar, lo que ocasionó que de acuerdo al Centro Estadístico de la UNESCO, el 95\% de los niños, niñas y adolescentes, esto es, el equivalente a 159 millones de alumnos en edad escolar en América Latina fueron afectados (UNICEF para América Latina y el Caribe, s.f.).

La crisis reveló las condiciones desfavorables y de desigualdad a la que están sometidos los alumnos de las instituciones escolares públicas, lo que generó que, en medio de esta nueva realidad, las instituciones escolares en todos los niveles educativos, se reorganizaran y adaptaran para responder a esta nueva demanda, surgen así, interrogantes como ¿Deben aprender lo mismo que de manera presencial? ¿Cómo garantizar aprendizaje significativo y formación integral en estas condiciones adversas? Elementos como la misión escolar, el perfil del alumno, del docente y del padre de familia, así como los ajustes al diseño curricular, se convierten en el centro de los nuevos direccionamientos a nivel organizacional.

Se parte de la necesidad de reflexionar la misión escolar, ya que a raíz de la aguda crisis de salud, su labor gira en torno a mejorar los sistemas de comunicación para motivar e integrar a todos los actores escolares a través de diferentes fuentes de contacto, promover la seguridad, el cuidado personal, la salud mental y reactivar la movilidad curricular ahora mediada por las TIC, liderar la reestructuración del diseño curricular hacia la pertinencia y la priorización de los aprendizajes, así como la reorientación de los proyectos pedagógicos para potencializar la actividad física y recreativa, el trabajo colaborativo en familia, la sana convivencia, el respeto, la tolerancia, la resiliencia; como estrategia de prevención de la violencia intrafamiliar y el abuso sexual, cuyos índices se dispararon a lo largo de la pandemia.

En este mismo orden de ideas, es indispensable de igual forma, replantear el perfil del alumno y del docente, ya que, en medio de esta nueva normalidad, el alumno adquiere un papel protagónico, más autónomo, consciente $\mathrm{y}$ responsable de su proceso de formación fortalecido en las habilidades blandas, tecnológicas, físico deportivas y con equilibrio mental y emocional. Se enfrenta a Ambientes Virtuales de Aprendizaje diseñados por los docentes para orientar su proceso de formación a través de plataformas educativas como Classroom y de comunicación sincrónica como Google Meet, zoom y Microsoft Teams, entre otras, las cuales enriquecen sus aprendizajes, ahora más alineados a su carácter de nativos digitales.

En cuanto al plan de estudios y la evaluación, urge su flexibilidad basándose en los principios de seguridad, equidad e inclusión, para adaptarse a los ritmos, necesidades, carencias y dificultades que presentan las familias para proveer las herramientas y materiales para el desarrollo de las clases propuestas ahora en línea o sincrónica y/o a través de guías pedagógicas, de tal forma que se pueda contrarrestar el desconcierto, desilusión y soledad en que se ve envuelto el alumno, minimizando el impacto de la deserción escolar.

Bajo este nuevo paradigma curricular, el docente es alumno y guía, ya que necesariamente empieza a adaptarse, reaprender cómo 
diseñar e implementar sus guías de aprendizaje en casa articuladas con objetos y ambientes virtuales de aprendizaje, manejar plataformas de comunicación, mensajería instantánea, en suma, potenciar sus habilidades tecnológicas; así como gestionar el volumen de información que recibe como actividades desarrolladas por los estudiantes para llevar un mejor control y poder retroalimentar de manera adecuada.

El docente en el marco de la pandemia, acelera la movilización de sus competencias en el uso pedagógico de las TIC para planear y desarrollar las clases y además ante las circunstancias de nuevas formas de comunicación, diseña e implementa diferentes formas de atender a los alumnos: uso de plataformas, video llamadas, llamadas, WhatsApp y guías impresas para los alumnos que no disponen de ningún tipo de conectividad. En el marco del contexto educativo virtual, adquiere pertinencia pedagógica el empleo de estrategias como el Aprendizaje Basado en Problemas, Aprendizaje Basado en Proyectos, Estudio de casos y el trabajo colaborativo.

Se destaca el papel de la familia como agente dinamizador, orientador del aprendizaje en casa, en medio de la semipresencialidad, virtualidad o aprendizaje remoto. Asume la familia un papel fundamental, siendo considerado un factor determinante para garantizar la permanencia y continuidad de los alumnos en la escuela.

La evaluación, es un proceso que permea el diseño curricular, da cuenta de la evolución de los niveles de progreso en el alcance de las competencias, en consecuencia, es de carácter formativa, pero a su vez, da cuenta de un producto y del nivel de impacto del diseño curricular; esto es, del Proyecto Educativo Institucio- nal en relación con el contexto sociocultural.

De este aspecto, se desprende un reto importante, movilizar las prácticas evaluativas frecuentes que dan cuenta de los contenidos desarrollados (evaluación sumativa) hacia las estrategias de evaluación formativa. El sistema de evaluación, debe estar alineado con las competencias planteadas, los aprendizajes esperados y los procesos de pensamiento potencializados, de tal forma que dé cuenta del nivel de avance de la competencia, de manera integral.

La evaluación en el contexto de la pandemia del COVID-19, toma su nivel de máxima flexibilidad, atendiendo a los ritmos y necesidades de los alumnos desde la diversidad de contextos situacionales en los que la escuela está circunscrita y a la cual sirve, esto es, adquiere la sensibilidad que en tales condiciones, la torna humanizada.

Por otro lado, en los diseños curriculares por competencias adquiere relevancia la movilización articulada e interrelacionada de diferentes tipos de conocimientos, capacidades y actitudes (contenidos cognitivos, procedimentales y actitudinales). Así, aparece el segundo reto, transformar los diseños curriculares, para que den respuesta al desarrollo de competencias, atendiendo a la articulación de los conocimientos relacionados entre áreas y/o asignaturas, y que además éstos respondan a las necesidades educativas contextuales; superando así los conocimientos aislados, descontextualizados y el gran volumen de áreas y/o asignaturas a desarrollar.

\section{Docencia e investigación}

La estrecha relación que existe entre la competencia pedagógica del docente y el logro $\mathrm{u}$ éxito escolar, exige el compromiso a ni- 
vel personal, profesional e institucional para promover el desarrollo profesional docente como elemento clave para potencializar los aprendizajes. Docentes íntegros y cualificados, capaces de mantener una interacción constante y asertiva con sus educandos, logrando con ello que el estudiante se enamorare, empoderarse del conocimiento a través de buenas prácticas pedagógicas.

En consecuencia, para fortalecer las competencias pedagógicas del docente, se sugiere que esté en un proceso de formación constante para manejar diferentes estrategias de aprendizaje, que faciliten y potencialicen el desarrollo de las competencias y en consecuencia pueda dar respuesta efectiva a los desafíos de la sociedad del conocimiento (Organziación para la Cooperación y el Desarrollo Económico, 2019). En esta perspectiva, cobra vigencia la diada indisoluble docencia - investigación, consideradas parte fundamental de la formación docente y de las transformaciones pedagógicas necesarias para alcanzar la eficiencia y eficacia en las prácticas áulicas.

La investigación, como estrategia pedagógica y metodológica, es ampliamente abordada por Malagón Plata, y otros (2019), destaca la capacidad de propiciar las transformaciones de los procesos pedagógicos, ya que fomenta la reflexión de la práctica pedagógica, esto es, se convierte en una oportunidad para su mejora, superando así las prácticas tradicionales. En otras palabras, resignifica el arte de enseñar para que responda a las características y necesidades contextuales de los estudiantes, aportando sentido e impactando positivamente en sus proyectos de vida.

En forma similar, se plantea que cambia el rol del docente, esto es, activa la par- ticipación del docente y los estudiantes en la indagación y búsqueda del conocimiento, siendo el docente un mediador del proceso. En este sentido, la investigación se convierte en una estrategia de innovación metodológica, didáctica y curricular, en la medida en que se construye y reconstruyen los planes de asignatura y fomenta la producción intelectual, a razón de compartir a través de artículos, ensayos, libros, sus reflexiones en torno a los avances en el desarrollo de las competencias, y a su vez, fortalecer el uso de las bases metodológicas de la investigación.

Podemos afirmar entonces, que al posicionar la investigación como elemento clave del desarrollo curricular, esta pasa a ser la propuesta para la acción transformadora, contribuyendo a la modernización institucional, ya que materializa los cambios en las prácticas académicas, administrativas y de gestión.

La práctica pedagógica concebida desde la reflexión a partir de la experimentación didáctica, no solo valida teorías, sino que genera aprendizajes teóricos, contribuyendo a la cultura del mejoramiento continuo de las prácticas de aula, a la eficiencia, eficacia y calidad educativa; y en consecuencia al mejoramiento institucional, en tanto se compartan las experiencias exitosas a través de las comunidades de aprendizaje. De acuerdo con esto, es posible plantear que la investigación pedagógica in situ transforma a los actores educativos de reproductores de teoría a generadores de conocimiento contextualizado, y se convierte en un reto, extender sustancialmente la práctica de la investigación pedagógica tradicionalmente desarrollada en los terrenos de la universidad, hacia el contexto escolar. 


\section{CONCLUSIONES}

La organización de la escuela sobre la cual se promueve las competencias para el siglo XXI, tiene como eje central el currículo, elemento que articula, cohesiona y contextualiza la política educativa, alrededor de las necesidades particulares de las comunidades, fundamentadas en prácticas profesionales conscientes, responsables y actualizadas. Lo que requiere formación continua del educa- dor en el campo disciplinar, didáctico pedagógico e investigativo; así mismo consolidar los nuevos escenarios educativos, de comunicación y de participación de los padres y las familias, los roles activos de los estudiantes y mediador del docente; ambientes de aprendizaje permeados y enriquecidos por las Tecnologías de la Información y las Comunicaciones, los cuales tomaron auge durante la pandemia del COVID-19; y se convierten en un reto al mantenerlos, en la pos pandemia.

\section{REFERENCIAS BIBLIOGRÁFICAS}

Coll, C. (2012). Las TIC, la nueva ecología del aprendizaje y la educación formal. Obtenido de https://www.youtube.com/watch?v=3-FRdOBbvnk

Fonseca Pérez, J., y Gamboa Graus, M. (2017). Aspectos teóricos sobre el diseño curricular y sus particularidades en las ciencias. Obtenido de file://D:/Downloads/Dialnet-AspectosTeoricosSobreElDisenoCurricularYSusParticu-6132044.pdf

Malagón Plata, L., Rodríguez Rodríguez , 1., Nañez Rodríguez, J., Obando Rodríguez, A., Villada Salazar, C., y Caicedo Duarte, C. (2019). Consolidación de la relación docencia-investigación en la educación superior (1a ed.). Ibagué: Universidad del Tolima. Obtenido de file://D:/Downloads/relaciones_docencia_e_investigacion_LIBRO.pdf

Organziación para la Cooperación y el Desarrollo Económico. (2019). El trabajo de la OCDE sobre educación y competencias. Obtenido de https:/www.oecd.org/education/El-trabajo-de-la-ocde-sobre-educacion-y-competencias.pdf

Pastrana Armínola, L., y Oviedo, P. (2014). Investigaciones y desafíos para la docencia del siglo XXI. Bogotá D.C: Kimpres Universidad De La Salle. Obtenido de http://biblioteca.clacso.edu.ar/Colombia/fce-unisalle/20170117032546/investigacion.pdf

Sacristán, J. (2010). ¿Qué significa el currículum? Obtenido de http://www.scielo.org.mx/scielo. php?script=sci_arttext\&pid=S1665-109X2010000100009

Santiváñez Limas, V. (2012). Diseño curricular a partir de competencias (segunda ed.). Bogotá: Ediciones de la U. ISBN 978-958-762-076-4 
UNESCO. (2013). Enfoques estratégicos sobre las TICS en la educación en América Latina y el Caribe. http://www.unesco.org/new/fileadmin/MULTIMEDIA/FIELD/Santiago/images/ ticsesp.pdf

UNICEF para América Latina y el Caribe. (s.f.). Covid-19: Preparación y respuesta educativa. https://www.unicef.org/lac/la-educacion-frente-al-covid-19 